\title{
An Elliptic Optimal Control Problem and its Two Relaxations*
}

\author{
Behrouz Emamizadeh \\ Faculty of Science and Engineering \\ The University of Nottingham Ningbo, China \\ Behrouz.Emamizadeh@ nottingham.edu.cn \\ Amin Farjudian \\ Center for Research on Embedded Systems \\ Halmstad University, Sweden \\ Amin.Farjudian@hh.se \\ Hayk Mikayelyan \\ Faculty of Science and Engineering \\ The University of Nottingham Ningbo, China \\ Hayk.Mikayelyan@ nottingham.edu.cn
}

July, 2016

\begin{abstract}
In this note, we consider a control theory problem involving a strictly convex energy functional, which is not Gâteaux differentiable. The functional came up in the study of a shape optimization problem, and here we focus on the minimization of this functional. We relax the problem in two different ways, and show that the relaxed variants can be solved by applying some recent results on two-phase obstacle like problems of free boundary type. We derive an important qualitative property of the solutions, i. e., we prove that the minimizers are three-valued, a result which significantly reduces the search space for the relevant numerical algorithms.
\end{abstract}

Key Words: Minimization, Free boundary, Optimality condition, Non-smooth analysis

Mathematics Subject Classification: 49J20, 35R35

\section{Introduction}

We consider the minimization of a functional, which has been studied in the context of two-phase membrane problem by several authors [1-3]. In [1], the relation between the minimization problem and optimal control theory has been mentioned.

The functional is strictly convex, but not Gâteaux differentiable, and we minimize the functional over suitable admissible sets. Using the recent results from the theory of free boundary problems, we prove that the solutions satisfy an important qualitative property. Specifically, we prove that the minimizers are three-valued, a result which reduces the search space for any numerical solution of the problem from a large function space to a more manageable space of three-valued functions.

In what follows, we present the formal statement of the main results in Section2. The tools and preliminary results form the content of Section 3, which is followed by the proofs of the two main theorems of the paper in Sections 4 and 5. Section 6 contains the conclusions.

${ }^{*}$ The final publication is available at link.springer.com and http://dx.doi.org/10.1007/s10957-016-0983-1 


\section{Statement of the Main Results}

As already mentioned, this note is concerned with the minimization of a strictly convex energy functional, which fails to be Gâteaux differentiable. The functional, $\Phi: W^{1,2}(D) \rightarrow \mathbb{R}$, is defined by:

$$
\Phi(u):=\frac{1}{2} \int_{D}|\nabla u|^{2} d x+\int_{D}|u| d x-\int_{\partial D} \psi u d \sigma,
$$

in which $D \subseteq \mathbb{R}^{N}$, and $\psi \in L^{2}(\partial D)$ satisfies $\int_{\partial D} \psi d \sigma=0$. We will consider the minimization problem over three admissible sets.

First, define $W:=\left\{u \in W^{1,2}(D): \int_{D} u d x=0\right\}$, and let:

$$
\begin{aligned}
& \mathcal{F}_{1}:=\left\{f \in L^{\infty}(D): \forall x \in D:-1 \leq f(x) \leq 1, \int_{D} f d x=0\right\}, \\
& \mathcal{F}_{2}:=\left\{f \in L^{\infty}(D): \sup _{x \in D} f-\inf _{x \in D} f \leq 2, \int_{D} f d x=0\right\} .
\end{aligned}
$$

Observe that $\mathcal{F}_{1} \subset \mathcal{F}_{2}$. For each $f \in \mathcal{F}_{2}$, we use the notation $S(f)$ to denote the set of solutions of the following Neumann boundary value problem:

$$
\begin{cases}-\Delta u=f & \text { in } D \\ \frac{\partial u}{\partial v}=\psi & \text { on } \partial D\end{cases}
$$

and we define:

$$
\left\{\begin{array} { r l } 
{ K _ { 1 } } & { : = \bigcup _ { f \in \mathcal { F } _ { 1 } } S ( f ) , } \\
{ P ( K _ { 1 } ) } & { : = W \cap K _ { 1 } , }
\end{array} \quad \left\{\begin{array}{rl}
K_{2} & :=\bigcup_{f \in \mathcal{F}_{2}} S(f), \\
P\left(K_{2}\right) & :=W \cap K_{2} .
\end{array}\right.\right.
$$

Remark 2.1. If we use the divergence theorem on 2.3 , we will get the compatibility condition $\int_{D} f d x=$ $-\int_{\partial D} \psi d \sigma$. As we had already assumed that $\int_{\partial D} \psi d \sigma=0$, we required $f$ to satisfy $\int_{D} f d x=0$ in the definitions of $\mathcal{F}_{1}$ and $\mathcal{F}_{2}$ in (2.1) and (2.2), respectively.

Remark 2.2. It is well known that the set $\mathcal{G}:=\left\{g \in L^{\infty}(D): 0 \leq g \leq 1, \int_{D} g d x=\alpha\right\}$ is the $\sigma\left(L^{\infty}, L^{1}\right)$-closure of the set

$$
\mathcal{G}^{\prime}:=\left\{\chi_{E}: E \text { is a measurable subset of } D \text { and }|E|=\alpha\right\},
$$

in which $\chi_{E}$ is the characteristic function of the set $E$, and $|E|$ is its $N$-dimensional Lebesgue measure. Here, $\sigma\left(L^{\infty}, L^{1}\right)$ denotes the $w^{*}$-topology on $L^{\infty}(D)$. A straightforward argument proves that $\mathcal{F}_{1}$ is the $\sigma\left(L^{\infty}, L^{1}\right)$ closure of the set

$$
\mathcal{F}_{1}^{\prime}:=\left\{\chi_{E}-\chi_{E^{c}}: E \text { is a measurable subset of } D \text { and }|E|=\frac{1}{2}|D|\right\} .
$$

The functions in $\mathcal{F}_{1}^{\prime}$ are $\{-1,1\}$-valued. As a result, they are sometimes referred to as 'bang-bang' functions. Interestingly, the minimizers of our problems are, in general, not bang-bang functions, and can have three values.

We are interested in the following three minimization problems:

$$
\begin{gathered}
\inf _{u \in P\left(K_{1}\right)} \Phi(u), \\
\inf _{u \in K_{1}} \Phi(u), \\
\inf _{u \in P\left(K_{2}\right)} \Phi(u) .
\end{gathered}
$$

Remark 2.3. Both problems 2.5) and 2.6) are relaxed versions of 2.4. In the case of 2.5, we relax the minimization problem (2.4) by extending the admissible set "orthogonally" to $W$, and get the cylindrical set $K_{1}$. In the case of 2.6, we extend the admissible set within $W$ as $P\left(K_{1}\right) \subset P\left(K_{2}\right) \subset W$. 
Our main results in this paper are the following two theorems:

Theorem 2.1. The minimization problem 2.5] has a unique solution $u_{0}$. Moreover:

(i) $\Delta u_{0}=\chi_{\left\{u_{0}>0\right\}}-\chi\left\{u_{0}<0\right\}$,

(ii) $\left|\left\{u_{0}<0\right\}\right|=\left|\left\{u_{0}>0\right\}\right|$,

where for any $X \subseteq \mathbb{R}^{N}$, the notation $|X|$ has been used for the $N$-dimensional Lebesgue measure of $X$.

Proof. See Section 4, page 5 .

Remark 2.4. Note that the assertions (ii) and (iii) in Theorem 2.1 imply that $u_{0}$ can be neither strictly positive nor strictly negative in the entire domain $D$.

Theorem 2.2. The minimization problem 2.6 has a unique solution $v_{0}$. Moreover, there exists a unique constant $\left.h_{0} \in\right]-1,1[$, such that:

(i) $\Delta v_{0}=\left(1+h_{0}\right) \chi\left\{v_{0}>0\right\}-\left(1-h_{0}\right) \chi\left\{v_{0}<0\right\}$,

(ii) $\left(1+h_{0}\right)\left|\left\{v_{0}>0\right\}\right|=\left(1-h_{0}\right)\left|\left\{v_{0}<0\right\}\right|$.

Proof. See Section 5 , page 7 .

It should be pointed out that the minimization problem $(2.5)$ is not an optimal control problem, where one minimizes a functional with respect to an admissible set of controllers and a partial differential state equation. Let us briefly explain this point. Suppose that $f \in \mathcal{F}_{1}$, and $u_{f}$ is the unique solution of the boundary value problem 2.3 for which $\int_{D} u_{f} d x=0$; thus, $u_{f} \in P\left(K_{1}\right) \subset K_{1}$. As a result, we obtain:

$$
\inf _{f \in \mathcal{F}_{1}} \Phi\left(u_{f}\right)=\inf _{u \in P\left(K_{1}\right)} \Phi(u) .
$$

If $\int_{D} u_{0} d x \neq 0$, where $u_{0}$ is the unique solution of 2.5$]$, then we have:

$$
\inf _{f \in \mathcal{F}_{1}} \Phi\left(u_{f}\right)=\inf _{u \in P\left(K_{1}\right)} \Phi(u)>\inf _{u \in K_{1}} \Phi(u) .
$$

The authors make the conjecture that the solution $w_{0}$ of the minimization problem (2.4) is the projection of $u_{0}$ onto $W$. In other words, if $\Phi\left(u_{0}\right)=\inf _{u \in K_{1}} \Phi(u)$, and

$$
w_{0}(x):=P\left(u_{0}\right)(x)=u_{0}(x)-\frac{1}{|D|} \int_{D} u_{0}(x) d x
$$

then $\Phi\left(w_{0}\right)=\inf _{v \in P\left(K_{1}\right)} \Phi(v)$.

Studying the minimization problems 2.5) and 2.6 was a natural task for us as we were attempting to construct solutions of the so-called two-phase obstacle like problem

$$
\Delta u=\lambda_{+} \chi_{\{u>0\}}-\lambda_{-} \chi_{\{u<0\}},
$$

where $\lambda_{ \pm}$are positive Lipschitz functions, which we needed in our study of a shape optimization problem. In [1], it has been proven that the free boundary of $\{u=0\}$ in a neighborhood of each branch point $x \in \partial\{u>$ $0\} \cap \partial\{u<0\} \cap\{\nabla u=0\}$ is a union of $C^{1}$ graphs (also, see [2, 3]). This result helps us in drawing significant qualitative conclusions about the optimal shapes. An effective numerical method is presented in [4].

In what follows, $\|\cdot\|_{p}$ denotes the usual $L^{p}$-norm, $\|\cdot\|_{p, \partial D}$ denotes the $L^{p}$-norm on the boundary of $D$, and $\|\cdot\|$ denotes the $W^{1,2}$-norm. Moreover, the symbol $C$ will indicate various constants at different stages with different values. 


\section{Preliminaries}

In this section, we collect some tools which will help us in proving Theorems 2.1 and 2.2 . We begin with the observation that $W=\left\{u \in W^{1,2}(D):\langle u, 1\rangle=0\right\}$, where $\langle\cdot, \cdot\rangle$ denotes the inner product in $W^{1,2}(D)$. Hence, we can write $W^{1,2}(D)$ as the direct sum $W^{1,2}(D)=W \bigoplus \mathbb{R}$. As a consequence, the projection $P: W^{1,2}(D) \rightarrow$ $W^{1,2}(D)$, with range $\mathcal{R}(P)=W$ and null set $\mathcal{N}(P)=\mathbb{R}$, is well defined, and we have:

$$
W^{1,2}(D)=\mathcal{R}(P) \bigoplus \mathcal{N}(P)
$$

Whence, every $u \in W^{1,2}(D)$ can be uniquely written as $u=v+c$, for some $v \in W$ and $c \in \mathbb{R}$.

Note that each $K \in\left\{K_{1}, K_{2}\right\}$ is a cylindrical set, in the sense that $K+\mathbb{R}=K$. This, in turn, implies that the projection $P(K)$ of $K$ is contained in $K$, and $K=P(K) \bigoplus \mathbb{R}$. Fig. 1 provides an intuitive picture.

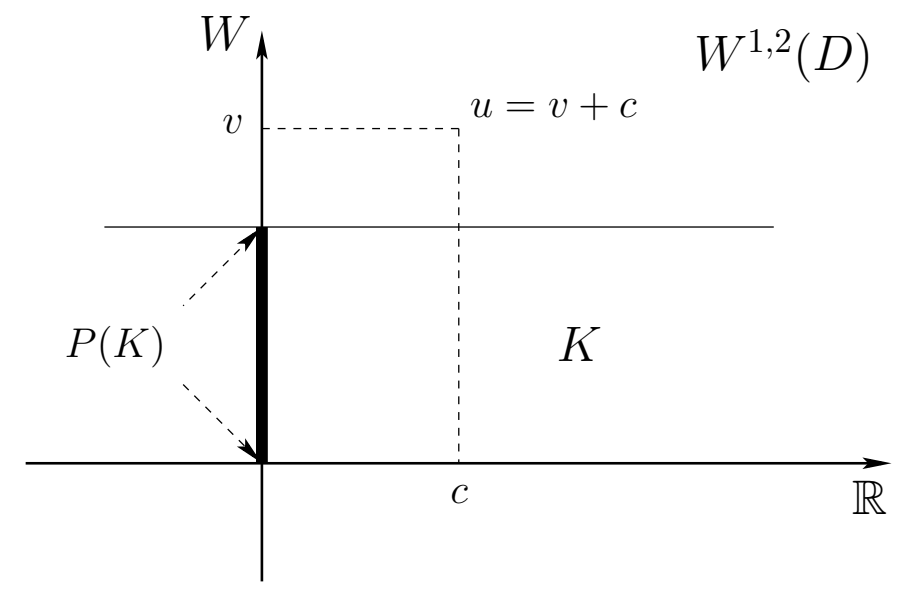

Figure 1: The set $W^{1,2}(D)$, and its cylindrical subset $K \in\left\{K_{1}, K_{2}\right\}$, can be written as the direct sums $W^{1,2}(D)=$ $W \bigoplus \mathbb{R}$ and $K=P(K) \bigoplus \mathbb{R}$, respectively.

Let us mention two more properties of $K_{1}$ and $K_{2}$ :

Lemma 3.1. The sets $K_{1}, P\left(K_{1}\right), K_{2}$, and $P\left(K_{2}\right)$, are convex and closed in $W^{1,2}(D)$.

Proof. First, note that both $\mathcal{F}_{1}$ and $\mathcal{F}_{2}$ are convex sets:

$$
\forall f, g \in \mathcal{F}_{1}\left(\text { or } \in \mathcal{F}_{2}\right), \lambda \in[0,1]: \lambda f+(1-\lambda) g \in \mathcal{F}_{1}\left(\text { or } \in \mathcal{F}_{2}\right) \text {. }
$$

This entails the convexity of $K_{1}$ and $K_{2}$ as for any $u_{1} \in S(f)$ and $u_{2} \in S(g)$, we have: $\lambda u_{1}+(1-\lambda) u_{2} \in$ $S(\lambda f+(1-\lambda) g)$.

To prove closedness of $K_{1}$, we consider a sequence $u_{n} \in K_{1}$ such that $u_{n} \rightarrow u$ in $W^{1,2}(D)$. We need to show that $u \in K_{1}$. Note that by definition, there exists a sequence $\left(f_{n}\right) \subseteq \mathcal{F}_{1}$ such that $\forall n: u_{n} \in S\left(f_{n}\right)$. Hence, the following integral equation holds for each $n$ :

$$
\int_{D} \nabla u_{n} \cdot \nabla w d x-\int_{\partial D} \psi w d \sigma-\int_{D} f_{n} w d x=0, \quad \forall w \in W^{1,2}(D) .
$$

Since $\left(f_{n}\right)$ is bounded in $L^{\infty}(D) \simeq\left(L^{1}(D)\right)^{*}$, we deduce that there is a subsequence—still denoted by $\left(f_{n}\right)$ - such that $f_{n} \rightarrow_{w^{*}} f$ in $L^{\infty}(D)$. By the Banach-Alaoglu theorem, $\mathcal{F}_{1}$ is $w^{*}$-compact, hence $w^{*}$-closed, and thereby $f \in \mathcal{F}_{1}$. Returning to 3.1 , and passing to the limit under the integrals, we obtain:

$$
\int_{D} \nabla u \cdot \nabla w d x-\int_{\partial D} \psi w d \sigma-\int_{D} f w d x=0, \quad \forall w \in W^{1,2}(D) .
$$

From (3.2) we deduce that $u \in S(f)$. Hence, $u \in K_{1}$.

Closedness of $K_{2}$ can be proved similarly. Closedness and convexity of $P\left(K_{1}\right)$ and $P\left(K_{2}\right)$ follow from the closedness and convexity of $W$. 
For the minimization problem 2.5 to make sense we need to make sure that $\Phi$ is bounded from below. In fact, it turns out that $\Phi$ is bounded from below throughout $W^{1,2}(D)$, not just on $K_{1}$ or $K_{2}$ :

Lemma 3.2. The functional $\Phi$ is bounded from below on $W^{1,2}(D)$.

Proof. Every $u \in W^{1,2}(D)$ can be written as $u=v+c$, for some $v \in W$ and $c \in \mathbb{R}$. Now, from the definition of $\Phi$ we obtain:

$$
\begin{aligned}
\Phi(u) & \geq \frac{1}{2} \int_{D}|\nabla v|^{2} d x-\|\psi\|_{2, \partial D}\|v\|_{2, \partial D} \\
\text { (trace embedding) } & \geq \frac{1}{2}\left(\|\nabla v\|_{2}^{2}-C\|\psi\|_{2, \partial D}\|v\|\right) \\
\text { (Poincaré) } & \geq \frac{1}{2}\left(\|\nabla v\|_{2}^{2}-C\|\psi\|_{2, \partial D}\|\nabla v\|_{2}\right) \\
& \geq \frac{1}{2}\left(\|\nabla v\|_{2}-\frac{C}{2}\|\psi\|_{2, \partial D}\right)^{2}-\frac{C^{2}}{8}\|\psi\|_{2, \partial D}^{2} \\
& \geq-\frac{C^{2}}{8}\|\psi\|_{2, \partial D}^{2} .
\end{aligned}
$$

Note that the trace embedding that we have used is of type $W^{1,2}(D) \rightarrow L^{2}(\partial D)$ (see, e.g., [5]). Clearly, inequality 3.3 shows that $\Phi$ is bounded from below, as claimed.

\section{Proof of Theorem 2.1}

Proof. We begin with a minimizing sequence $\left(u_{n}\right) \subseteq K_{1}$. Since $u_{n}=v_{n}+c_{n}$ for $v_{n} \in P\left(K_{1}\right)$ and $c_{n} \in \mathbb{R}$, and $\int_{\partial D} \psi d \sigma=0$, we have:

$$
\Phi\left(u_{n}\right)=\frac{1}{2} \int_{D}\left|\nabla v_{n}\right|^{2} d x+\int_{D}\left|v_{n}+c_{n}\right| d x-\int_{\partial D} \psi v_{n} d \sigma
$$

Note that:

$$
\begin{aligned}
\int_{D}\left|v_{n}+c_{n}\right| d x & \geq \int_{D}\left|c_{n}\right| d x-\int_{D}\left|v_{n}\right| d x \\
& =\left|c_{n}\right||D|-\left\|v_{n}\right\|_{1} \\
\left(L^{2} \hookrightarrow L^{1}\right) & \geq\left|c_{n}\right||D|-C_{1}\left\|v_{n}\right\|_{2} \\
\text { (Poincaré and } \left.\int_{D} v_{n} d x=0\right) & \geq\left|c_{n}\right||D|-C_{2}\left\|\nabla v_{n}\right\|_{2},
\end{aligned}
$$

for some constants $C_{1}$ and $C_{2}$. Thus, using the trace embedding, Poincaré inequality, and the fact that $\int_{D} v_{n} d x=$ 0 , from (4.1) and (4.2) we infer:

$$
\Phi\left(u_{n}\right) \geq \frac{1}{2}\left\|\nabla v_{n}\right\|_{2}^{2}+\left|c_{n}\right||D|-C\left\|\nabla v_{n}\right\|_{2},
$$

for some constant $C$. Inequality 4.3 , together with Lemma 3.2, implies that the real sequences $\left(\left\|\nabla v_{n}\right\|_{2}\right)$ and $\left(c_{n}\right)$ are bounded. Thus, there exist $v_{0} \in W$ and $c_{0} \in \mathbb{R}$ such that for a subsequence-still denoted $\left(u_{n}\right)$-we have $u_{n} \rightarrow u_{0}=v_{0}+c_{0}$ in $W^{1,2}(D)$. By Lemma 3.1, the set $K_{1}$ is closed and convex. Thus, it is weakly closed and $u_{0} \in K_{1}$. Due to the compact embedding $W^{1,2}(D) \hookrightarrow L^{2}(D)$, a subsequence—still denoted by $\left(u_{n}\right)$ —converges to $u_{0}$ almost everywhere in $D$. As a result, we have:

(1) $\left\|\nabla v_{0}\right\|_{2} \leq \liminf _{n \rightarrow \infty}\left\|\nabla v_{n}\right\|_{2}$, as $W^{1,2}$-norm is weakly lower semi-continuous.

(2) $\int_{D}\left|u_{0}\right| d x \leq \liminf _{n \rightarrow \infty} \int_{D}\left|u_{n}\right| d x$, by Fatou's Lemma. 
(3) $\lim _{n \rightarrow \infty} \int_{\partial D} \psi v_{n} d \sigma=\int_{\partial D} \psi v_{0} d \sigma$.

From (1), (2), and (3), we infer that $\Phi\left(u_{0}\right) \leq \liminf _{n \rightarrow \infty} \Phi\left(u_{n}\right)$, which implies that $u_{0}$ solves the minimization problem 2.5. As $\Phi$ is strictly convex, $u_{0}$ is unique.

Interestingly, $u_{0}$ is also the minimizer of $\Phi$ over the whole space $W^{1,2}(D)$. In fact, the solution $u_{*}$ of the minimization problem

$$
\inf _{u \in W^{1,2}(D)} \Phi(u)
$$

is the solution of the so-called two-phase obstacle like problem with Neumann boundary data (see [3]):

$$
\begin{cases}\Delta u_{*}=\chi_{\left\{u_{*}>0\right\}}-\chi_{\left\{u_{*}<0\right\}} & \text { in } D, \\ \frac{\partial u_{*}}{\partial v}=\psi & \text { on } \partial D .\end{cases}
$$

The right-hand sides of (4.5) must satisfy the compatibility condition

$$
\int_{D} f_{*} d x=\int_{\partial D} \psi d \sigma=0
$$

for Neumann boundary value problems, where $f_{*}=-\chi_{\left\{u_{*}>0\right\}}+\chi_{\left\{u_{*}<0\right\}}$. Moreover, $-1 \leq f_{*} \leq 1$; thus, $f_{*} \in \mathcal{F}_{1}$ and $u_{*} \in K_{1}$. This means that $u_{*}=u_{0}$.

Theorem 2.1 (iii) follows from 4.6).

Remark 4.1. The solution $u_{0}$ of the minimization problem 2.5 satisfies the differential equation $-\Delta u_{0}=f_{0}$, where $-f_{0}=\chi_{\left\{u_{0}>0\right\}}-\chi_{\left\{u_{0}<0\right\}}$ is the right-hand side in the differential equation in (4.5). Whence, by local elliptic regularity theory $[6], u_{0} \in W_{l o c}^{2, p}(D)$, for every $\left.p \in\right] 1, \infty[$. In particular, we deduce that the equation holds almost everywhere in $D$. By applying Lemma 7.7 in [7], we infer that the function $f_{0}$ must vanish on flat sections of the graph of $u_{0}$. Thus, $f_{0}$ must vanish on the set $\left\{u_{0}=0\right\}$. This, in turn, implies that when $\left|\left\{u_{0}=0\right\}\right|$ is positive, $f_{0}$ cannot be an element of $\mathcal{F}_{1}^{\prime}$, as introduced in Remark 2.2. In other words, the function $f_{0}$ may not be a bang-bang function, but a three-valued one.

Remark 4.2. The optimality condition (4.5) can also be derived from $0 \in \partial \Phi\left(u_{0}\right)$. Let us first decompose $\Phi$ as $\Phi=\Phi_{1}+\Phi_{2}-\Phi_{3}$, in which $\Phi_{1}(u):=\frac{1}{2} \int_{D}|\nabla u|^{2} d x, \Phi_{2}(u):=\int_{D}|u| d x$, and $\Phi_{3}(u):=\int_{\partial D} \psi u d \sigma$. The functions $\Phi_{1}$ and $\Phi_{3}$ are Gâteaux differentiable:

$$
\partial_{w} \Phi_{1}\left(u_{0}\right)=\int_{D} \nabla u_{0} \cdot \nabla w d x, \quad \forall w \in W^{1,2}(D),
$$

and

$$
\partial_{w} \Phi_{3}\left(u_{0}\right)=\int_{\partial D} \psi w d \sigma, \quad \forall w \in W^{1,2}(D) .
$$

However, $\Phi_{2}$ is not globally Gâteaux differentiable as its directional derivative at any point $u$ in the direction of $w$ is:

$$
\partial_{w} \Phi_{2}(u)=\int_{\{u \neq 0\}} \operatorname{sgn}(u) w d x+\int_{\{u=0\}}|w| d x,
$$

where

$$
\operatorname{sgn}(x):=\left\{\begin{array}{cc}
1 & (x>0) \\
0 & (x=0) \\
-1 & (x<0)
\end{array}\right.
$$

Hence, at any $u$, the functional $\Phi_{2}$ is Gâteaux differentiable only if $|\{u=0\}|=0$. Therefore, $a$ priori, it is not known whether $\Phi_{2}$ - and as a result $\Phi$-will be Gâteaux differentiable at $u_{0}$ or not.

The functional $\Phi_{2}$, though not Gâteaux differentiable, is Lipschitz in $W^{1,2}(D)$, as:

$$
\left|\Phi_{2}\left(u_{1}\right)-\Phi_{2}\left(u_{2}\right)\right|=\left|\left\|u_{1}\right\|_{1}-\left\|u_{2}\right\|_{1}\right| \leq\left\|u_{1}-u_{2}\right\|_{1} \leq C\left\|u_{1}-u_{2}\right\|,
$$


for some constant $C$. Therefore, the optimality condition satisfied by $u_{0}$ is:

$$
0 \in \partial \Phi\left(u_{0}\right)+\mathcal{N}_{K}\left(u_{0}\right)
$$

where $\mathcal{N}_{K}\left(u_{0}\right)$ denotes the normal cone at $u_{0}$ supported on $K$. Since $K$ is convex (Lemma 3.1), we infer that $\mathcal{N}_{K}\left(u_{0}\right)$ coincides with $\partial \xi_{K}\left(u_{0}\right)$, in which $\xi_{K}$ denotes the indicator function supported on $K$ (see, e. g., [8,9]):

$$
\xi_{K}(w):= \begin{cases}0, & w \in K, \\ +\infty, & w \notin K,\end{cases}
$$

and

$$
\partial \xi_{K}\left(u_{0}\right)=\left\{g \in W^{1,2}(D): \xi_{K}(u) \geq \xi_{K}\left(u_{0}\right)+\left\langle g, u-u_{0}\right\rangle, \forall u \in W^{1,2}(D)\right\} .
$$

On the other hand:

$$
\partial \Phi\left(u_{0}\right) \subseteq \partial \Phi_{1}\left(u_{0}\right)+\partial \Phi_{2}\left(u_{0}\right)-\partial \Phi_{3}\left(u_{0}\right),
$$

since $-\Phi_{3}$ is convex [8]. Whence, from (4.9] and 4.11] we obtain:

$$
\nabla \Phi_{1}\left(u_{0}\right)+\gamma-\nabla \Phi_{3}\left(u_{0}\right)+g=0 \quad \text { in } W^{-1,2}(D),
$$

for some $\gamma \in \partial \Phi_{2}\left(u_{0}\right)$ and $g \in \partial \xi_{K}\left(u_{0}\right)$. From (4.12) we obtain:

$$
\begin{aligned}
& \left\langle\nabla \Phi_{1}\left(u_{0}\right), u-u_{0}\right\rangle+\left\langle\gamma, u-u_{0}\right\rangle \\
& -\left\langle\nabla \Phi_{3}\left(u_{0}\right), u-u_{0}\right\rangle+\left\langle g, u-u_{0}\right\rangle=0, \quad \forall u \in W^{1,2}(D) .
\end{aligned}
$$

At this stage, we use the fact that for convex and Lipschitz functionals $\partial \Phi$ coincides with subdifferential (see Propositions 2.1.5 and 2.2.7 in [8]), to get:

$$
\left\langle\gamma, u-u_{0}\right\rangle \leq \partial_{u-u_{0}} \Phi_{2}\left(u_{0}\right), \quad \forall u \in W^{1,2}(D) .
$$

Now, from 4.13, 4.14, and 4.10, we deduce:

$$
\begin{aligned}
\int_{D} \nabla u_{0} \cdot\left(\nabla u-\nabla u_{0}\right) d x-\int_{\partial D} \psi\left(u-u_{0}\right) d \sigma & \\
& +\int_{\left\{u_{0} \neq 0\right\}} \operatorname{sgn}\left(u_{0}\right)\left(u-u_{0}\right) d x+\int_{\left\{u_{0}=0\right\}}\left|u-u_{0}\right| d x \geq 0,
\end{aligned}
$$

for every $u \in K$. Note that in (4.15) we have used $\xi_{K}(u)=\xi_{K}\left(u_{0}\right)=0$, because both $u$ and $u_{0}$ are in $K$. Equation (4.5) can be derived from (4.15), for which we refer the interested reader to [3].

\section{Proof of Theorem 2.2}

Proof. As $\int_{D} u d x=0$ for $u \in W$, over $P\left(K_{2}\right) \subset W$, minimization of the functional

$$
\Phi(u)=\frac{1}{2} \int_{D}|\nabla u|^{2} d x+\int_{D}|u| d x-\int_{\partial D} \psi u d \sigma
$$

is equivalent to minimization of the functional

$$
\Phi_{h}(u):=\frac{1}{2} \int_{D}|\nabla u|^{2} d x+\int_{D}(|u|+h u) d x-\int_{\partial D} \psi u d \sigma,
$$

where $h \in[-1,1]$ is a parameter. Furthermore, we observe that the functional $\Phi_{h}$ can be written as:

$$
\Phi_{h}(u)=\frac{1}{2} \int_{D}|\nabla u|^{2} d x+\int_{D}\left((1+h) u^{+}+(1-h) u^{-}\right) d x-\int_{\partial D} \psi u d \sigma .
$$


Let us now denote by $u_{h}$ the unique solution of the minimization problem

$$
\inf _{u \in W^{1,2}(D)} \Phi_{h}(u),
$$

which is the solution of the following two-phase obstacle-like problem (see [3]):

$$
\begin{cases}\Delta u_{h}=(1+h) \chi_{\left\{u_{h}>0\right\}}-(1-h) \chi_{\left\{u_{h}<0\right\}} & \text { in } D, \\ \frac{\partial u_{h}}{\partial v}=\psi & \text { on } \partial D .\end{cases}
$$

In what follows, we will prove three claims, which will lead to the existence of a unique $\left.h_{0} \in\right]-1,1[$, such that $u_{h_{0}} \in W$. This entails that $v_{0}:=u_{h_{0}}$ is the unique solution of the minimization problem 2.6.

Claim 1. For all $x \in D: u_{1}(x) \leq 0$ and $u_{-1}(x) \geq 0$.

Proof. The function $u_{1}$ is the minimizer of the functional

$$
\Phi_{1}(u)=\frac{1}{2} \int_{D}|\nabla u|^{2} d x+\int_{D} 2 u^{+} d x-\int_{\partial D} \psi u d \sigma .
$$

If $M:=\sup _{x \in D} u_{1}(x)>0$, then the function $\tilde{u}:=u_{1}-M$ will have a smaller energy, i. e., $\Phi_{1}(\tilde{u})<\Phi_{1}\left(u_{1}\right)$, which is a contradiction. The proof of $u_{-1} \geq 0$ is similar.

Claim 2. If $h_{1}>h_{2}$, then for all $x \in D: u_{h_{1}}(x) \leq u_{h_{2}}(x)$.

Proof. Let us assume that $D_{*}:=\left\{x \in D: u_{h_{1}}(x)>u_{h_{2}}(x)\right\} \neq \emptyset$, and take:

$$
\left\{\begin{array}{l}
v_{1}:=\min \left(u_{h_{1}}, u_{h_{2}}\right), \\
v_{2}:=\max \left(u_{h_{1}}, u_{h_{2}}\right) .
\end{array}\right.
$$

We have $\Phi_{h_{1}}\left(u_{h_{1}}\right)<\Phi_{h_{1}}\left(v_{1}\right)$ and $\Phi_{h_{2}}\left(u_{h_{2}}\right)<\Phi_{h_{2}}\left(v_{2}\right)$. Adding up the two inequalities, and canceling out the repeating terms on both sides, we obtain:

$$
\begin{aligned}
\int_{D_{*}}\left(1+h_{1}\right) u_{h_{1}}^{+}+\left(1-h_{1}\right) u_{h_{1}}^{-}+\left(1+h_{2}\right) u_{h_{2}}^{+}+\left(1-h_{2}\right) u_{h_{2}}^{-} d x< & \\
& \int_{D_{*}}\left(1+h_{1}\right) u_{h_{2}}^{+}+\left(1-h_{1}\right) u_{h_{2}}^{-}+\left(1+h_{2}\right) u_{h_{1}}^{+}+\left(1-h_{2}\right) u_{h_{1}}^{-} d x,
\end{aligned}
$$

which can be written as:

$$
\begin{aligned}
& \left(h_{1}-h_{2}\right) \int_{D_{*}} u_{h_{1}} d x=\int_{D_{*}}\left(h_{1}-h_{2}\right)\left(u_{h_{1}}^{+}-u_{h_{1}}^{-}\right) d x< \\
& \quad \int_{D_{*}}\left(h_{1}-h_{2}\right)\left(u_{h_{2}}^{+}-u_{h_{2}}^{-}\right) d x=\left(h_{1}-h_{2}\right) \int_{D_{*}} u_{h_{2}} d x .
\end{aligned}
$$

This is a contradiction.

Claim 3. The mapping $h \mapsto u_{h}$ is continuous in $W^{1,2}(D)$. 
Proof. Assume that $h_{n} \rightarrow h_{0}$. Without loss of generality, we can assume that $h_{n}$ is monotone. The uniform convexity, coercivity, and lower semi-continuity of the family of functionals $\Phi_{h_{n}}$ makes it possible to find a convergent sub-sequence $u_{n} \rightarrow v$. In fact, because of monotonicity by the previous claim, we do not need to take a sub-sequence.

Evidently, $\Phi_{h_{0}}\left(u_{h_{0}}\right) \leq \Phi_{h_{0}}(v)=\lim _{n \rightarrow \infty} \Phi_{h_{n}}\left(u_{n}\right)$. On the other hand, from continuity of $\Phi_{h}$ with respect to $h$ and $u$, it follows that:

$$
\Phi_{h_{0}}\left(u_{h_{0}}\right) \geq \liminf _{n \rightarrow \infty} \Phi_{h_{n}}\left(u_{n}\right)=\Phi_{h_{0}}(v),
$$

since otherwise $\Phi_{h_{n}}\left(u_{0}\right)<\Phi_{h_{n}}\left(u_{n}\right)$ for $n$ large enough. The convexity of $\Phi_{h_{0}}$ and uniqueness of its minimizer yield that $u_{0}=v$.

The three aforementioned claims prove the existence of the minimizer and item (ii) of the theorem. Item (ii) follows from the fact that the integral of $\Delta v_{0}$ vanishes.

\section{Conclusions}

Many optimization algorithms rely on the existence of gradient, which in the context of what we have presented in this paper, translates into Gâteaux differentiability. The functional that we considered lacks this property, which makes any attempt at searching through the entire admissible function space extremely inefficient.

Yet, we proved that searching through the entire space is unnecessary as the solutions are bound to be three-valued. This reduces the search space significantly, and although the traditional gradient methods are not applicable, it is feasible to make use of other optimization methods to tackle the problem numerically.

\section{References}

[1] Shahgholian, H., Uraltseva, N., Weiss, G.S.: The two-phase membrane problem-regularity of the free boundaries in higher dimensions. International Mathematics Research Notices 2007 (2007)

[2] Emamizadeh, B., Prajapat, J.V., Shahgholian, H.: A two phase free boundary problem related to quadrature domains. Potential Analysis 34(2), 119-138 (2010)

[3] Petrosyan, A., Shahgholian, H., Uraltseva, N.: Regularity of free boundaries in obstacle-type problems, Graduate Studies in Mathematics, vol. 136. American Mathematical Society, Providence, RI (2012)

[4] Bozorgnia, F.: Numerical solutions of a two-phase membrane problem. Appl. Numer. Math. 61(1), 92-107 (2011)

[5] Adams, R.A., Fournier, J.J.F.: Sobolev spaces, Pure and Applied Mathematics, vol. 140, second edn. Elsevier/Academic Press, Amsterdam (2003)

[6] Agmon, S.: The $L_{p}$ approach to the Dirichlet problem. Part I : regularity theorems. Annali della Scuola Normale Superiore di Pisa - Classe di Scienze 13(4), 405-448 (1959)

[7] Gilbarg, D., Trudinger, N.S.: Elliptic Partial Differential Equations of Second Order. Springer-Verlag (2001)

[8] Clarke, F.H.: Optimization and Nonsmooth Analysis. Classics in Applied Mathematics. Society for Industrial and Applied Mathematics (1990)

[9] Ekeland, I., Temam, R.: Convex Analysis and Variational Problems, Studies in Mathematics and its Applications, vol. 1. North-Holland Publishing Co., Amsterdam-Oxford (1976). Translated from French 